\title{
Polarization difference between hyperons and antihyperons induced by an external magnetic field
}

\author{
Hai-Bo $\mathrm{Li}^{*}$ and Xin-Xin $\mathrm{Ma}^{\dagger}$ \\ Institute of High Energy Physics, Beijing 100049, People's Republic of China \\ and University of Chinese Academy of Sciences, Beijing 100049, People's Republic of China
}

(Received 25 August 2019; published 11 October 2019)

\begin{abstract}
We investigate the quantum correlated $\Lambda \bar{\Lambda}$ production in the reaction $e^{+} e^{-} \rightarrow J / \psi \rightarrow \Lambda \bar{\Lambda}$. Since the $\Lambda$ or $\bar{\Lambda}$ has a nonzero magnetic moment, its spin will undergo a Larmor precession in the magnetic field of the detector, such as the BESIII experiment. Because of the spin precession, the angular distribution of the $\Lambda$ and $\bar{\Lambda}$ is slightly modified. Therefore, we obtain the corresponding term of the modified angular distribution due to the effect of the Larmor precession. We also estimate its potential effect on the measurements of $C P$ violation, as well as the decay asymmetry parameter and polarization of $\Lambda$. The polarization of the $\Lambda$ or $\bar{\Lambda}$ at the production vertex will rotate around the $B$-field axis, over an angle depending on the flight length, but it still could be measured by fit to the corrected angular distribution. Of important note, we conclude that a nonzero $C P$ asymmetry of order $10^{-4}$ will be caused once neglecting spin precession of the $\Lambda$ and $\bar{\Lambda}$ in the $e^{+} e^{-} \rightarrow J / \psi \rightarrow \Lambda \bar{\Lambda}$ process. The size of this $C P$ asymmetry is several times that of predicted within Standard Model in the hyperon decay. Although this effect is small, it will play an important role in future high precision experiments, such as the super-taucharm factory.
\end{abstract}

DOI: $10.1103 /$ PhysRevD.100.076007

\section{INTRODUCTION}

Since the inner parts of a proton were found [1], probing the structure of baryons is still active. However, a complete observation of the electromagnetic (e.m.) structure of hadrons is possible merely in polarization experiments. The results for elastic scattering were first presented by the SLAC scattering experiments [2], in which both electrons and proton target are polarized. The electron-positron collider provides coherent hyperon-antihyperon pairs. In 2019, the BESIII experiment has collected about $10^{10} \mathrm{~J} / \psi$ decay events, which is an ideal place to probe the form factors and search for the $C P$ violation in the coherent $\Lambda \bar{\Lambda}$ pair production [3]. Recently, the most precision asymmetry parameter of $\Lambda$ is measured to be $0.750 \pm 0.009 \pm$ 0.004 [4], with more than $7.0 \sigma$ deviation from previous world averaged value [5]. Another determination of $\alpha_{-}$has also been presented, based on data from the SLAS collaboration [6], with more than $2.0 \sigma$ deviation from the BESIII's result. The BESIII detector consists mainly

\footnotetext{
lihb@ihep.ac.cn

†maxx@ihep.ac.cn
}

Published by the American Physical Society under the terms of the Creative Commons Attribution 4.0 International license. Further distribution of this work must maintain attribution to the author(s) and the published article's title, journal citation, and DOI. Funded by SCOAP ${ }^{3}$. of a cylindrical main draft chamber, with a magnetic field of $1.0 \mathrm{~T}$ parallel to the electron beam [3]. The $\Lambda$ and $\bar{\Lambda}$ are produced in the $e^{+} e^{-}$collision point, due to the long lifetime of hyperon, they will decay in flight and the average decay length or flight length will be $12 \mathrm{~cm}$ in the $B$ field $(1.0 \mathrm{~T})$ of the BESIII detector. Therefore, the hyperon will undergo a Larmor precession in the magnetic field in the detector. However, this effect was not considered in previous publications [4,6-11], which may account for the difference of the measurement of $\alpha_{-}$. This effect is small but very important, because the measurements of $C P$ violation could reach high sensitivity. The sensitivity of $C P$ violation in the charged- $\Xi$ decay has reached order $10^{-4}[12]$. In the future high precision experiments, such as the proposed super-tau-charm factory [13], the sensitivities on the $C P$ measurements will reach $10^{-4}$ or even $10^{-5}$. In those cases, one has to consider the spin precession effect, which will modify the angular distribution of the $e^{+} e^{-} \rightarrow$ $J / \psi \rightarrow \Lambda \bar{\Lambda}$ process, therefore the $C P$ asymmetry $A_{C P}=$ $\frac{\alpha_{-}+\alpha_{+}}{\alpha_{-}-\alpha_{+}}$will be biased, and nonzero $A_{C P}$ will be extracted once neglecting the Larmor precession. The paper is divided into two parts. In the first part, we will consider this effect and derive the corresponding modification on overall angular distributions of the final states. In the second part, we perform a Monte-Carlo (MC) simulation and then give the impact on the measurements of $\alpha_{ \pm}$and the $C P$ asymmetry parameters. 


\section{THE PRODUCTION OF $\Lambda \bar{\Lambda}$ PAIRS}

The coherent $\Lambda \bar{\Lambda}$ pairs are produced via the process $e^{+} e^{-} \rightarrow J / \psi \rightarrow \Lambda \bar{\Lambda}$. The $\Lambda$ and $\bar{\Lambda}$ with intrinsic magnetic moment will undergo a Larmor precession in the external magnetic field of the detector, so the spin direction will be changed in the flight before its decaying. This effect will modify the angular distribution of the process $e^{+} e^{-} \rightarrow J / \psi \rightarrow \Lambda \bar{\Lambda}$. The effective amplitude for $e^{+} e^{-} \rightarrow$ $J / \psi \rightarrow \Lambda \bar{\Lambda}$ can be written as

$$
\begin{aligned}
M= & \frac{i e^{2}}{q^{2}} j_{\mu} \bar{u}\left(p_{1}, s_{1}\right)\left(F_{1}^{H}\left(q^{2}\right) \gamma_{\mu}+\frac{F_{2}^{H}\left(q^{2}\right)}{2 m_{\Lambda}} p_{\nu} \sigma^{\nu \mu} \gamma_{5}\right) \\
& \times \nu\left(p_{2}, s_{2}\right),
\end{aligned}
$$

where $p_{1}\left(p_{2}\right)$ is the momenta of $\Lambda(\bar{\Lambda}), m_{\Lambda}$ the mass of $\Lambda$, $s_{1}\left(s_{2}\right)$ the spin four-vectors of $\Lambda(\bar{\Lambda}), q=p_{1}+p_{2}$, $p=p_{1}-p_{2}$, and $s=p^{2}, j_{\mu}=\bar{u}\left(k_{1}\right) \gamma_{\mu} \nu\left(k_{2}\right)$ is the lepton current with $k_{1}\left(k_{2}\right)$ the momenta of $e^{-}\left(e^{+}\right)$, Of important, the form factors $G_{E}^{H}$ and $G_{M}^{H}$ are usually called as hadronic form factors [14], because the $\Lambda \bar{\Lambda}$ are produced via the $J / \psi$ hadronic decay [15], are related to $F_{1,2}^{H}$ by

$$
G_{M}^{H}=F_{1}^{H}+F_{2}^{H}, \quad G_{E}^{H}=F_{1}^{H}+\tau F_{2}^{H},
$$

with $\tau=\frac{q^{2}}{4 m_{\Lambda}^{2}}$. Following the method in Refs. [16-18], the differential cross section takes the following form with all constants dropped

$$
\begin{aligned}
\frac{d \sigma}{d \cos \theta} \sim 1 & +\alpha_{\psi} \cos ^{2} \theta+\sin ^{2} \theta \hat{s}_{1}^{x} \hat{s}_{2}^{x}+\alpha_{\psi} \sin ^{2} \theta \hat{s}_{1}^{y} \hat{s}_{2}^{y} \\
& -\left(\alpha_{\psi}+\cos ^{2} \theta\right) \hat{s}_{1}^{z} \hat{s}_{2}^{z}+\sqrt{1-\alpha_{\psi}^{2}} \cos \Phi \sin \theta \\
& \times \cos \theta\left(\hat{s}_{1}^{x} \hat{s}_{2}^{z}-\hat{s}_{1}^{z} \hat{s}_{2}^{x}\right)+\sqrt{1-\alpha_{\psi}^{2}} \sin \Phi \sin \theta \\
& \times \cos \theta\left(\hat{s}_{1}^{y}-\hat{s}_{2}^{y}\right),
\end{aligned}
$$

where $\theta$ is the angle between momenta of $e^{+}$and $\Lambda$, $\alpha_{\psi}=\frac{\tau\left|G_{M}\right|^{2}-\left|G_{E}\right|^{2}}{\tau\left|G_{M}\right|^{2}+\left|G_{E}\right|^{2}}, \hat{s}_{1}^{i} \quad\left(\hat{s}_{2}^{i}\right)$ the $i$ th component of the unit vector pointing to the direction of the $\Lambda(\bar{\Lambda})$ spin in the rest frame of its mother particle with the $\mathrm{Z}$-axis direction defined by $\Lambda(\bar{\Lambda})$ momentum direction and Y-axis direction defined by $\vec{p}_{1} \times \vec{k}_{2}\left(\vec{p}_{2} \times \vec{k}_{2}\right)$. $\Phi$ is the relative phase of form factors,

$$
\frac{G_{E}^{H}}{G_{M}^{H}}=e^{i \Phi}\left|\frac{G_{E}^{H}}{G_{M}^{H}}\right|
$$

\section{III. $\Lambda$ SPIN PRECESSION}

Considering the interaction between the $\Lambda$ and the external magnetic field of the BESIII detector, we can easily obtain [19]

$$
\hat{s}_{1}^{\prime}=\hat{s}_{1}+\omega \tau_{\Lambda} \hat{B} \times \hat{s}_{1},
$$

where $\hat{s}_{1}^{\prime}$ denote the spin direction of $\Lambda$ in its rest frame at decay time $\tau_{\Lambda}$ since produced, $\hat{B}$ the direction of magnetic field in the $\Lambda$ rest frame, $\omega$ the precession frequency which depends on the magnetic field magnitude $B$ and the magnetic moment of $\Lambda$, can be written as

$$
\omega=-\frac{2 \mu_{\Lambda} B}{\hbar}
$$

where the $\mu_{\Lambda}$ is the magnetic moment of $\Lambda$ with the world average value $-0.613 \pm 0.04 \mu_{N}$ [5]. If one takes $B=1 T$, the lifetime of $\Lambda \tau_{\Lambda}=2.632 \times 10^{-10} \mathrm{~s}$, and the momentum of $\Lambda$ is about $1 \mathrm{GeV} / c$ in the rest frame of $J / \psi$, the average precession angle can be determined to be about $\mathcal{A}_{\text {rota }}=\omega \tau_{\Lambda}=0.017 \mathrm{rad}$, which will potentially contribute to the decay parameters measurement. Similarly, for other hyperons, $\Xi, \Omega, \Sigma^{ \pm}$, the spin precession should also be considered.

After considering this effect, the spin direction of $\Lambda$ became $\hat{s}_{1}^{\prime}$ when it decays in flight, so the decay amplitude of $\Lambda \rightarrow p \pi^{-}$could be written as

$$
\left|M_{1}\right|^{2} \sim 1+\alpha_{-} \hat{s}_{1}^{\prime} \cdot n_{p}
$$

where $\alpha_{-}$is the decay parameter of $\Lambda$, as well as $\alpha_{+}$the decay parameter for $\bar{\Lambda}, n_{p}$ the flight direction of proton in the hyperon rest frame. Usually the $C P$ asymmetry is defined as $A_{C P}=\frac{\alpha_{-}+\alpha_{+}}{\alpha_{-}-\alpha_{+}}$. Recently, the $A_{C P}$ is measured to be $A_{C P}=-0.006 \pm 0.012 \pm 0.007$ [4], while the theoretical prediction within the SM is order $10^{-5}[20,21]$.

After undergoing a spin precession in the magnetic field $\vec{B}$, at the decay time $\tau_{\Lambda}$, the spin direction of $\Lambda$ becomes

$$
\left(\begin{array}{l}
\hat{s}_{1}^{\prime x} \\
\hat{s}_{1}^{\prime y} \\
\hat{s}_{1}^{\prime z}
\end{array}\right)=\left(\begin{array}{lll}
1 & -\mathcal{A}_{\text {rota }} \hat{B}_{z}^{\prime} & \mathcal{A}_{\text {rota }} \hat{B}_{y}^{\prime} \\
\mathcal{A}_{\text {rota }} \hat{B}_{z}^{\prime} & 1 & -\mathcal{A}_{\text {rota }} \hat{B}_{x}^{\prime} \\
-\mathcal{A}_{\text {rota }} \hat{B}_{y}^{\prime} & \mathcal{A}_{\text {rota }} \hat{B}_{x}^{\prime} & 1
\end{array}\right)\left(\begin{array}{l}
\hat{s}_{1}^{x} \\
\hat{s}_{1}^{y} \\
\hat{s}_{1}^{z}
\end{array}\right),
$$

where $\hat{B}^{\prime}=\hat{B}+(\gamma-1)\left(\hat{B} \cdot n_{\Lambda}\right) n_{\Lambda}$ with $n_{\Lambda}$ the flight direction of $\Lambda$ in the rest frame of $J / \psi$ [22]. Then we will average the spin of $\Lambda$, and apply the relationship

$$
\left\langle\hat{s}^{i} \hat{s}^{j}\right\rangle=\delta^{i j}
$$

Then we will obtain the total differential cross section for the full decay chain, in which the $\Lambda(\bar{\Lambda})$ decay into $p \pi^{-}$ $\left(\bar{p} \pi^{+}\right)$. Here what we need to do is to replace $\left(s_{1}^{x}, s_{1}^{y}, s_{1}^{z}\right)$ with 


$$
\left(\begin{array}{lll}
1 & \mathcal{A}_{\text {rota }} \hat{B}_{z}^{\prime} & -\mathcal{A}_{\text {rota }} \hat{B}_{y}^{\prime} \\
-\mathcal{A}_{\text {rota }} \hat{B}_{z}^{\prime} & 1 & \mathcal{A}_{\text {rota }} \hat{B}_{x}^{\prime} \\
\mathcal{A}_{\text {rota }} \hat{B}_{y}^{\prime} & -\mathcal{A}_{\text {rota }} \hat{B}_{x}^{\prime} & 1
\end{array}\right)\left(\begin{array}{l}
\alpha_{-} n_{p}^{x} \\
\alpha_{-} n_{p}^{y} \\
\alpha_{-} n_{p}^{z}
\end{array}\right),
$$

so as $s_{2}$. Then the differential cross section can be obtained as where the $\Omega_{1,2}$ is the solid angle of proton and antiproton in the

$$
\begin{aligned}
\frac{d \sigma}{d \cos \theta d \Omega_{1} d \Omega_{2}} \sim & 1+\alpha_{\psi} \cos ^{2} \theta+\sin ^{2} \theta \alpha_{-} \alpha_{+} n_{p}^{x} n_{\bar{p}}^{x}+\alpha_{\psi} \alpha_{-} \alpha_{+} \sin ^{2} \theta n_{p}^{y} n_{\bar{p}}^{y}-\left(\alpha_{\psi}+\cos ^{2} \theta\right) \alpha_{-} \alpha_{+} n_{p}^{z} n_{\bar{p}}^{z}+\sqrt{1-\alpha_{\psi}^{2}} \\
& \times \alpha_{-} \alpha_{+} \cos \Phi \sin \theta \cos \theta\left(n_{p}^{x} n_{\bar{p}}^{z}-n_{p}^{z} n_{\bar{p}}^{x}\right)+\sqrt{1-\alpha_{\psi}^{2}} \sin \Phi \times \sin \theta \cos \theta\left(\alpha_{-} n_{p}^{y}-\alpha_{+} n_{\bar{p}}^{y}\right) \\
& +\alpha_{-} \alpha_{+} \mathcal{A}_{\text {rota }} \sin ^{2} \theta\left(\hat{B}_{z}^{\prime}\left(\hat{n}_{\bar{p}}^{x} \hat{n}_{p}^{y}-\hat{n}_{p}^{x} \hat{n}_{\bar{p}}^{y}\right)-\hat{B}_{y}^{\prime}\left(\hat{n}_{p}^{x} \hat{n}_{\bar{p}}^{z}+\hat{n}_{\bar{p}}^{x} \hat{n}_{p}^{z}\right)\right) \\
& +\alpha_{\psi} \alpha_{-} \alpha_{+} \mathcal{A}_{\text {rota }} \sin ^{2} \theta\left(-\hat{B}_{z}^{\prime} \hat{n}_{p}^{x} \hat{n}_{\bar{p}}^{y}+\hat{B}_{z}^{\prime} \hat{n}_{\bar{p}}^{x} \hat{n}_{p}^{y}-\hat{B}_{x}^{\prime} \hat{n}_{p}^{y} \hat{n}_{\bar{p}}^{z}+\hat{B}_{x}^{\prime} \hat{n}_{\bar{p}}^{y} \hat{n}_{p}^{z}\right) \\
& -\alpha_{+} \alpha_{-} \mathcal{A}_{\text {rota }}\left(\alpha_{\psi}+\cos ^{2} \theta\right)\left(\hat{B}_{y}^{\prime} \hat{n}_{p}^{x} \hat{n}_{\bar{p}}^{z}-\hat{B}_{x}^{\prime} \hat{n}_{p}^{y} \hat{n}_{\bar{p}}^{z}+\hat{B}_{y}^{\prime} \hat{n}_{\bar{p}}^{x} \hat{n}_{p}^{z}+\hat{B}_{x}^{\prime} \hat{n}_{\bar{p}}^{y} \hat{n}_{p}^{z}\right) \\
& +\mathcal{A}_{\text {rota }} \alpha_{-} \alpha_{+} \sqrt{1-\alpha_{\psi}^{2}} \cos \Phi \sin \theta \cos \theta\left(\hat{B}_{x}^{\prime} \hat{n}_{p}^{x} \hat{n}_{\bar{p}}^{y}+\hat{B}_{x}^{\prime} \hat{n}_{\bar{p}}^{x} \hat{n}_{p}^{y}+\hat{B}_{z}^{\prime} \hat{n}_{p}^{y} \hat{n}_{\bar{p}}^{z}+\hat{B}_{z}^{\prime} \hat{n}_{\bar{p}}^{y} \hat{n}_{p}^{z}\right) \\
& +\mathcal{A}_{\text {rota }} \sqrt{1-\alpha_{\psi}^{2}} \sin \Phi \sin \theta \cos \theta \times\left(\alpha_{+} \hat{B}_{x}^{\prime} \hat{n}_{\bar{p}}^{z}-\alpha_{-} \hat{B}_{z}^{\prime} \hat{n}_{p}^{x}+\alpha_{-} \hat{B}_{x}^{\prime} \hat{n}_{p}^{z}-\alpha_{+} \hat{B}_{z}^{\prime} \hat{n}_{\bar{p}}^{x}\right),
\end{aligned}
$$

rest frame of $\Lambda$ and $\bar{\Lambda}$, respectively. We should notice that the spin precession could modify the $\Lambda$ polarization state

$$
\begin{aligned}
& P_{\Lambda}^{x}=-\mathcal{A}_{\text {rota }} \hat{B}_{z}^{\prime} P_{\Lambda}^{y} \\
& P_{\Lambda}^{z}=\mathcal{A}_{\text {rota }} \hat{B}_{x}^{\prime} P_{\Lambda}^{y},
\end{aligned}
$$

where the $P_{\Lambda}^{x, y, z}$ denote the polarization projection on the $x$, $y$, and $z$ axis. The $P_{\Lambda}^{x, z}$ must be zero if there is no spin precession, as shown in Fig. 1.

\section{MONTE CARLO SIMULATION AND RESULTS}

Because the spin precession is usually neglected or missed in the current experimental studies, the MC simulation is essential for numerical study on the effect of spin precession. The parameters $\alpha_{\psi}, \Phi$, and $\alpha_{ \pm}$are set according to the measurement result in Ref. [4]. Exactly, we take $\alpha_{\psi}=0.462, \Phi=0.738$ and $\alpha_{ \pm}= \pm 0.750$ assuming no $C P$ violation. In the BESIII experiment, the magnitude of

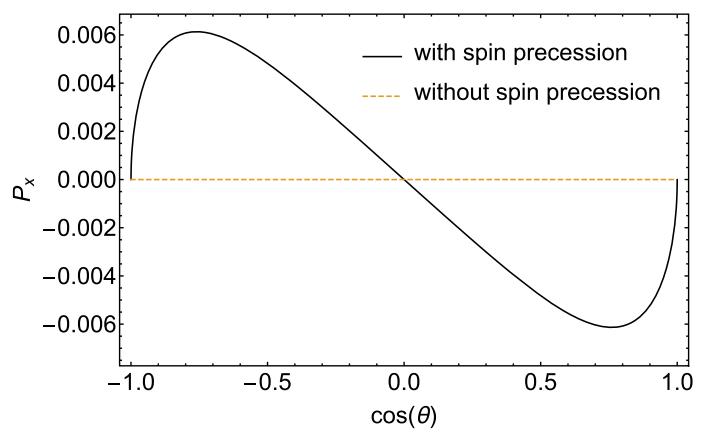

FIG. 1. The solid black and dashed orange lines denote the $P_{\Lambda}^{y}$ of $\Lambda$ with and without spin precession effect, respectively. the magnetic field is around 1T. The lifetime of the $\Lambda$ that depends on its momentum also strongly affects the precession angle. Here we take the momentum of $\Lambda$ at $\sqrt{s / 4-m^{2}}$ with $\sqrt{s}$ collider energy $3.097 \mathrm{GeV}$.

The MC simulation is performed based on ROOT [23]. First the phase space events are generated, then an acceptance-rejection method is adopted to get the signal toy MC samples based on the distribution in Eq. (11).

To reveal the effect on the measurement of the parameters $\alpha_{\psi}, \Phi$, and $\alpha_{ \pm}$, especially the $A_{C P}$, we perform the maximum likelihood fit to the toy $\mathrm{MC}$ samples.

The probability distribution function is defined as

$$
\mathcal{P}=\frac{1}{N} \frac{\mathrm{d} \sigma}{\mathrm{d} \cos \theta \mathrm{d} \Omega_{1} \mathrm{~d} \Omega_{2}},
$$

where $N$ is the normalization factor which is determined to be $(4 \pi)^{2}\left(1+\alpha_{\psi} / 3\right)$. The likelihood is defined as

$$
-\ln \mathcal{L}=-\sum_{i=1}^{n} \ln \mathcal{P}_{i}
$$

where $i$ denotes the $i$ th events in the MC sample, $n$ is the total number of events in the MC sample which is set at $1 \times 10^{6}$. The fitted value of the parameter with Eq. (11) is defined as $\alpha_{ \pm}^{\text {truth }}$. Then we remove this effect, in which the precession frequency is just fixed at zero so that the Eq. (11) will be same as Eq. (3), then fit to the same toy MC sample again, the fitted value of decay parameters is referred as $\alpha_{ \pm}^{\text {biased }}$. The differences between the results of the two fits are

$$
\Delta \alpha_{ \pm}=\alpha_{ \pm}^{\text {biased }}-\alpha_{ \pm}^{\text {truth }}
$$




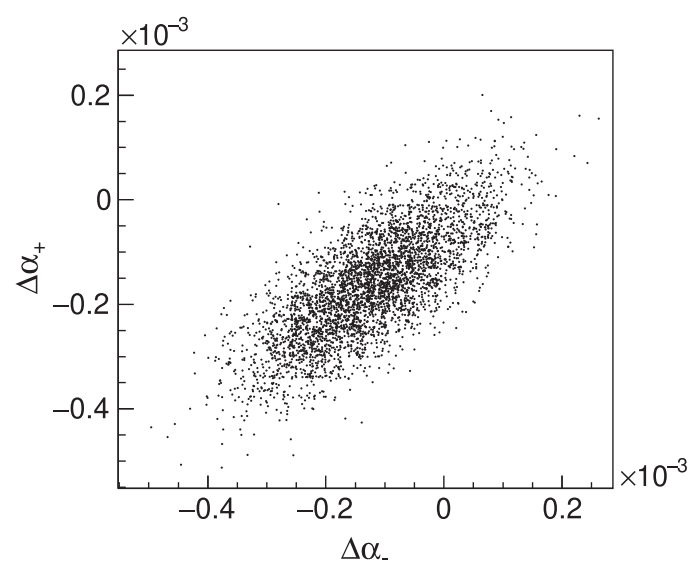

FIG. 2. The strongly correlation between $\Delta \alpha_{-}$and $\Delta \alpha_{+}$. Each black point denotes the result from fitting to each toy $\mathrm{MC}$ sample.

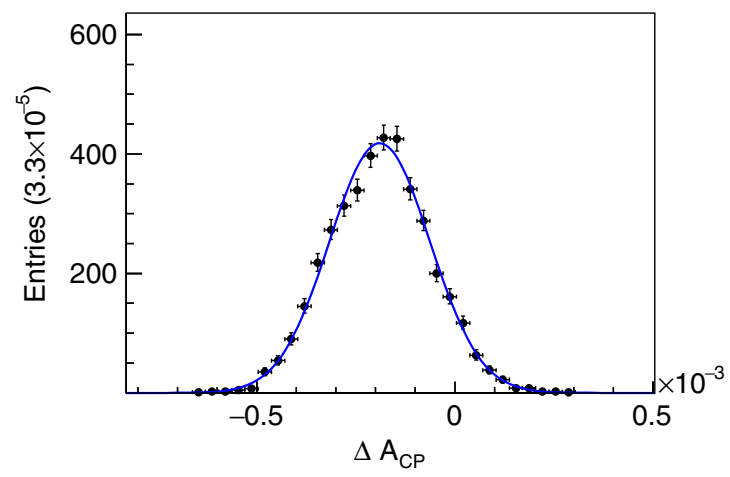

(a)

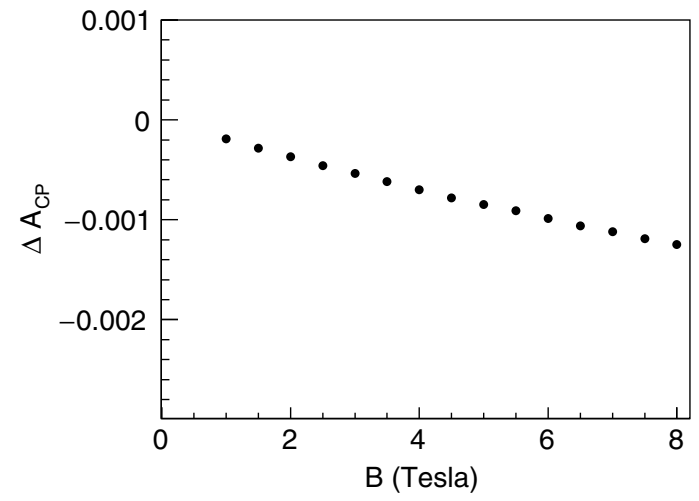

(b)

FIG. 3. (a) The distribution of $\Delta A_{C P}$ from 4000 times fits to the toy $\mathrm{MC}$ sample. The average of $\Delta A_{C P}$ and corresponding uncertainty are determined by fit to the distribution with a Gaussian function. (b) The $\Delta A_{C P}$ will deviated more from zero, if the magnitude of the external magnetic field is increasing.

We generate 4000 toy MC samples with the same data size, and find that the strong correlation between $\Delta \alpha_{-}$and $\Delta \alpha_{+}$as shown in Fig. 2. This strong correlation leads to

$$
\begin{aligned}
\Delta A_{C P} & =\frac{1}{n} \sum_{i=1}^{n} \frac{\Delta \alpha_{-, i}+\Delta \alpha_{+, i}}{\alpha_{-, i}^{\text {biased }}-\alpha_{+, i}^{\text {biased }}} \\
& =(-1.9 \pm 0.1) \times 10^{-4},
\end{aligned}
$$

where $i$ denotes the fit result from the $i$ th toy MC sample, $n$ the number of total fit results, and take $\alpha_{-}^{\text {truth }}+\alpha_{+}^{\text {truth }}=0$ where $C P$ conservation is assumed. The size of $\Delta A_{C P}$ is several times that of the $A_{C P}$ predicted by the $\mathrm{SM}$, as shown in Fig. 3(a). What is more, as we expect, the larger the magnitude of the magnitude field $B$ is, the farther off zero the corresponding $\Delta A_{C P}$ will be, as shown in Fig. 3(b). The values of $\alpha_{\psi}$ and $\Phi$ will also be derived from the truth values relatively about $0.07 \%$ and $0.01 \%$, respectively when the spin precession is neglected in the experiment.

\section{SUMMARY}

In this work, we considered the spin precession of hyperon in the magnetic field of the detector and gave the differential cross section for the global decay chain. The corrected term is proportional to the lifetime of $\Lambda$ and the magnetic field. The effects of spin precession ware also estimated, based on the MC simulation. The polarization of $\Lambda$ will be changed. We found that a deviation of order $10^{-4}$ on the $C P$ asymmetry will be induced once neglecting the spin precession, which is the same level as that from the SM prediction. As well, a small deviation of $\alpha_{\psi}$ and $\Phi$ will be caused due to this effect. The effect of the Larmor precession of hyperon in the external magnetic field has been also studied in Refs. [24-26]. Following the method in this work, the effect could be easily extended to other hyperon pair production at BESIII, such as $\Xi \bar{\Xi}, \Sigma^{0} \bar{\Sigma}^{0}, \Omega \bar{\Omega}$, etc., In the further, the super-tau-charm factor will reach a sensitivity of $10^{-4}$ or even $10^{-5}$ [13], we suggest that one should consider the effect due to spin precession of hyperons, so that one can determine the value of $\alpha_{ \pm}$and $C P$ asymmetry correctly in the experiment.

\section{ACKNOWLEDGMENTS}

The authors especially thank H.-n. Li, Mao-Zhi Yang, and Xian-Wei Kang for useful discussions. This work is supported in part by the National Natural Science Foundation of China under Contracts No. 11335009, No. 11125525, No. 11675137, No. 11875054, No. 11935018, the Joint Large-Scale Scientific Facility Funds of the NSFC and CAS under Contract No. U1532257, CAS under Contract No. U1632107, CAS under Contract No. QYZDJ-SSW-SLH003, and the National Key Basic Research Program of China under Contract No. 2015CB856700. 
[1] E. E. Chambers and R. Hofstadter, Structure of the proton, Phys. Rev. 103, 1454 (1956).

[2] M. J. Alguard et al., Elastic Scattering of Polarized Electrons by Polarized Protons, Phys. Rev. Lett. 37, 1258 (1976).

[3] H. B. Li, Prospects for rare and forbidden hyperon decays at BESIII, Front. Phys. (Beijing) 12, 121301 (2017); Erratum, Front. Phys. (Beijing) 14, 64001(E) (2019).

[4] M. Ablikim et al. (BESIII Collaboration), Polarization and entanglement in baryon-antibaryon pair production in electron-positron annihilation, Nat. Phys. 15, 631 (2019).

[5] M. Tanabashi et al. (Particle Data Group), Review of Particle Physics*, Phys. Rev. D 98, 030001 (2018).

[6] D. Ireland, M. Döring, D. Glazier, J. Haidenbauer, M. Mai, R. Murray-Smith, and D. Rönchen, Kaon Photoproduction and the $\Lambda$ Decay Parameter $\alpha_{-}$, arXiv:1904.07616 [Phys. Rev. Lett. (to be published)].

[7] M. Ablikim et al. (BESIII Collaboration), Study of $J / \psi$ and $\psi(3686)$ decay to $\Lambda \bar{\Lambda}$ and $\Sigma^{0} \bar{\Sigma}^{0}$ final states, Phys. Rev. D 95, 052003 (2017).

[8] M. Ablikim et al. (BES Collaboration), Study of $J / \psi$ decays to $\Lambda \bar{\Lambda}$ and $\Sigma^{0} \bar{\Sigma}^{0}$, Phys. Lett. B 632, 181 (2006).

[9] B. Aubert et al. (BABAR Collaboration), Study of $e^{+} e^{-} \rightarrow \Lambda \bar{\Lambda}, \Lambda \bar{\Sigma}^{0}, \Sigma^{0} \bar{\Sigma}^{0}$ using initial state radiation with $B A B A R$, Phys. Rev. D 76, 092006 (2007).

[10] M. Ablikim et al. (BESIII Collaboration), First observation of the isospin violating decay $J / \psi \rightarrow \Lambda \bar{\Sigma}^{0}+c . c$., Phys. Rev. D 86, 032008 (2012).

[11] B. Aubert et al. (BABAR Collaboration), Measurement of the branching fraction and anti-Lambda polarization in $B^{0} \rightarrow \bar{\Lambda} p \pi^{-}$, Phys. Rev. D 79, 112009 (2009).

[12] T. Holmstrom et al. (HyperCP Collaboration), Search for $C P$ Violation in Charged- $\Xi$ and $\Lambda$ Hyperon Decays, Phys. Rev. Lett. 93, 262001 (2004).

[13] I. I. Bigi, X. W. Kang, and H. B. Li, CP asymmetries in strange baryon decays, Chin. Phys. C 42, 013101 (2018).
[14] G. Faldt, Polarization observables in the $e^{+} e^{-} \rightarrow \bar{\Lambda} \Lambda$ reaction, Eur. Phys. J. A 52, 141 (2016).

[15] G. Faldt and A. Kupsc, Hadronic structure functions in the $e^{+} e^{-} \rightarrow \bar{\Lambda} \Lambda$ reaction, Phys. Lett. B 772, 16 (2017).

[16] A. Z. Dubnickova, S. Dubnicka, and M. P. Rekalo, Investigation of the nucleon electromagnetic structure by polarization effects in $e^{+} e^{-} \rightarrow N \bar{N}$ processes, Nuovo Cimento Soc. Ital. Fis. 109A, 241 (1996).

[17] G. I. Gakh and E. Tomasi-Gustafsson, General analysis of polarization phenomena in $e^{+}+e^{-} \rightarrow N+\bar{N}$ for axial parametrization of two-photon exchange, Nucl. Phys. A771, 169 (2006).

[18] H. Czyz, A. Grzelinska, and J. H. Kuhn, Spin asymmetries and correlations in lambda-pair production through the radiative return method, Phys. Rev. D 75, 074026 (2007).

[19] J. J. Sakurai and J. Napolitano, Modern Quantum Physics (Addison-Wesley, Reading, MA, 2011).

[20] J. F. Donoghue, X. G. He, and S. Pakvasa, Hyperon decays and CP nonconservation, Phys. Rev. D 34, 833 (1986).

[21] J. Tandean and G. Valencia, CP violation in hyperon nonleptonic decays within the standard model, Phys. Rev. D 67, 056001 (2003).

[22] L. D. Landau and E. M. Lifshitz, The Classical Theory of Fields (Pergamon, Oxford, 1951).

[23] R. Brun and F. Rademakers, ROOT-An object oriented data analysis framework, Nucl. Instrum. Methods Phys. Res., Sect. A 389, 81 (1997).

[24] D. E. Kharzeev, J. Liao, S. A. Voloshin, and G. Wang, Chiral magnetic and vortical effects in high-energy nuclear collisions-A status report, Prog. Part. Nucl. Phys. 88, 1 (2016).

[25] X. G. Deng and Y. G. Ma, Magnetic field effects on photon emission in intermediate energy heavy-ion collisions, Eur. Phys. J. A 54, 204 (2018).

[26] Y. Guo, S. Shi, S. Feng, and J. Liao, Magnetic field induced polarization difference between hyperons and anti-hyperons, https://dx.doi.org/10.1016/j.physletb.2019.134929. 